\title{
Post-streptococcal uveitis syndrome in a Caucasian population: a case series
}

\author{
David S. Curragh ${ }^{1} \cdot$ Clara E. McAvoy $^{1} \cdot$ Madeleine Rooney $^{2} \cdot$ Eibhlin McLoone $^{1}$
}

Received: 24 April 2018 / Accepted: 22 August 2018 / Published online: 18 September 2018

(c) The Royal College of Ophthalmologists 2018

\begin{abstract}
Background/Objectives Uveitis is an uncommon manifestation of post-streptococcal syndrome (PSUS). Despite reports, the condition is often not well recognised. The purpose of this study is to report a case series of children with post-streptococcal uveitis.

Subjects/Methods In this retrospective case series, all cases of PSUS were identified from all new paediatric patients diagnosed with uveitis over a 6-year period. Diagnosis of PSUS was based on the following diagnostic criteria: unilateral or bilateral uveitis with positive anti-streptolysin O titres (ASOT) or anti-deoxyribonuclease (anti-DNase) titres, and negative routine investigations for other causes of uveitis.

Results Eleven Caucasian paediatric patients were diagnosed with PSUS. One had a novel finding of peripheral corneal endotheliopathy, $73 \%$ of patients presented in spring or winter months and $88 \%$ of eyes had a final VA of better than or equal to 6/12 at a mean follow-up of 22 months. Systemic immunosuppressant treatment was used in $36 \%$ of patients. Adalimumab was used in $18 \%$ of patient's refractory to other treatment.

Conclusions We report on the largest consecutive series of Caucasian patients under 16 years of age with PSUS. We have demonstrated a seasonal preponderance with presentation typically in winter or spring. We report a novel finding of corneal endotheliopathy in one of our PSUS patients. We also report on the benefit of adalimumab in the management of severe cases of PSUS; use of biologics in this particular cohort of uveitis patients has not previously been reported. With aggressive treatment our patients achieved good visual outcomes comparable to other published series.
\end{abstract}

\section{Introduction}

Beta-haemolytic streptococci are a common cause of acute infections such as upper respiratory infection or impetigo. Immune-mediated complications are most commonly seen 7-35 days post infection with rheumatic fever reported in $0.7-1.4 \%$ of cases and glomerulonephritis in $0.2-3.4 \%$ of cases [1]. Uveitis is an uncommon manifestation of poststreptococcal syndrome. It was first reported in 1991 by Cokington and Han [2] when they presented a case of uveitis occurring 2 weeks after streptococcal pharyngitis.

David S. Curragh

davidcurragh@hotmail.com

1 Department of Ophthalmology, Royal Victoria Hospital, 274 Grosvenor Road, BT12 6BA Belfast, UK

2 Centre for Experimental Medicine, Queens University of Belfast, BT9 7BL Belfast, UK
Despite further reports in the published literature, the condition is often not well recognised [3-16]. The purpose of this study is to report a case series of children with poststreptococcal uveitis, to identify a spectrum of clinical features common to post-streptococcal syndrome (PSUS) and to report on visual outcomes, complications and treatments.

\section{Materials and methods}

Data were collected retrospectively from charts of all uveitis patients attending the Belfast Paediatric Uveitis Regional Service which commenced in April 2011 within the Belfast Health and Social Care Trust, Northern Ireland. All cases of PSUS were identified from all new patients diagnosed between April 2011 and March 2017. We adopted the same diagnostic criteria for PSUS employed by Tinley et al. [5] in the largest reported case series to date which was based on a South African population; i.e., the presence of unilateral or 
Table 1 Individual patient characteristics

\begin{tabular}{|c|c|c|c|c|c|c|c|c|c|c|c|c|}
\hline Case & Season & Initial VA & Lateral & PS & Posterior & $\begin{array}{l}\text { Anti- } \\
\text { DNase }\end{array}$ & ASOT & ESR & Topical & Systemic & Episodes & Final VA \\
\hline 1 & Spring & $6 / 66 / 6$ & Bilateral & - & - & - & 800 & 2 & $\mathrm{Y}$ & - & 1 & $6 / 66 / 6$ \\
\hline 2 & Spring & $6 / 38$ & Unilateral & - & $\begin{array}{l}\text { CMO, } \\
\text { papillitis }\end{array}$ & 800 & 800 & 2 & $\mathrm{Y}$ & Pred, MTX, adalimumab & 1 & $6 / 19$ \\
\hline 3 & Autumn & $6 / 12$ & Unilateral & - & - & 100 & 800 & 2 & $\mathrm{Y}$ & - & 1 & $6 / 9$ \\
\hline 4 & Winter & $6 / 7.5$ & Unilateral & $\mathrm{Y}$ & Papillitis & 200 & 800 & 7 & $\mathrm{Y}$ & - & 1 & $6 / 6$ \\
\hline 5 & Autumn & $6 / 36$ & Unilateral & - & $\begin{array}{l}\text { CMO, } \\
\text { vitreous haem }\end{array}$ & 400 & 800 & 2 & $\mathrm{Y}$ & $\begin{array}{l}\text { Pred, MTX, } \\
\text { mycophenolate, } \\
\text { adalimumab }\end{array}$ & 1 & $6 / 18$ \\
\hline 6 & Winter & $6 / 9$ & Unilateral & $\mathrm{Y}$ & - & - & 800 & 12 & $\mathrm{Y}$ & - & 2 & $6 / 5$ \\
\hline 7 & Spring & $6 / 6$ 6/6 & Bilateral & - & - & 1200 & 400 & 2 & $\mathrm{Y}$ & - & 1 & $6 / 66 / 4$ \\
\hline 8 & Winter & $6 / 12$ & Unilateral & $\mathrm{Y}$ & - & 1200 & 200 & 6 & $\mathrm{Y}$ & - & 1 & $6 / 6$ \\
\hline 9 & Winter & $6 / 56 / 9$ & Bilateral & - & - & 200 & 1600 & 100 & $\mathrm{Y}$ & MTX & 1 & $6 / 56 / 6$ \\
\hline 10 & Spring & $6 / 66 / 6$ & Bilateral & $\mathrm{Y}$ & Cataract & - & 400 & 34 & $\mathrm{Y}$ & MTX & 1 & $6 / 56 / 5$ \\
\hline 11 & Winter & $6 / 66 / 6$ & Bilateral & - & - & 600 & 200 & 8 & $\mathrm{Y}$ & - & 1 & $6 / 56 / 5$ \\
\hline
\end{tabular}

$V A$ visual acuity, $P S$ posterior synechiae, $C M O$ cystoid macular oedema, haem haemorrhage, ASOT anti-streptolysin O titres, anti-DNase antideoxyribonuclease titres,

ESR erythrocyte sedimentation rate, Pred prednisolone, MTX methotrexate

bilateral uveitis with positive anti-streptolysin $\mathrm{O}$ titres (ASOT) or anti-deoxyribonuclease (anti-DNase) titres, and negative routine investigations for other causes of uveitis [5]. Uveitis was classified according to the Standardisation of Uveitis Nomenclature (SUN) classification of the International Uveitis Study group based on the location of the inflammation [17]. Demographics, disease characteristics, treatments and complications were recorded. Visual acuity (VA) was measured at baseline, at 1- and 3-year time points and at final visit. Final visit was recorded where follow-up longer than 3 years had occurred, but for cases diagnosed less than 3 years, the visual acuity at last examination was included. Remission was defined as inactive disease for 3 months after discontinuing all treatments for uveitis [17]. Institutional board approval for the study was obtained via the Belfast Health and Social Care Trust's Standards, Quality and Audit department.

\section{Results}

\section{Demographics}

There were 11 patients with PSUS onset before the age of 16 years within the population studied over a 6 -year period from April 2011 to March 2017. This represented 11.6\% of all cases of paediatric uveitis seen at the Belfast Paediatric Uveitis Regional Service. The patients are listed in Table 1 and their demographics are summarised in Table 2. The mean follow-up was 22 months ranging from 3 to 56 months. One patient was lost to follow-up. The mean age
Table 2 Summary of patient characteristics

\begin{tabular}{ll}
\hline No. of patients & 11 \\
Gender & \\
Male & $18 \%$ \\
Female & $82 \%$ \\
Ethnicity & \\
Caucasian & $100 \%$ \\
Mean age at diagnosis & 11 Years 8 months \\
$0-2$ & $0 \%$ \\
$2-10$ & $36 \%$ \\
$10-16$ & $64 \%$ \\
Location & \\
Anterior & $55 \%$ \\
Intermediate & $45 \%$ \\
Posterior & $0 \%$ \\
Panuveitis & $0 \%$ \\
Laterality & \\
Bilateral & \\
Unilateral & $45 \%$ \\
Course & $55 \%$ \\
Acute & \\
Chronic & $18 \%$ \\
Recurrent & $73 \%$ \\
\hline
\end{tabular}

at presentation was 11 years and 8 months with age of presentation ranging from 8.5 to 15 years of age.

Of particular interest is case 5 (Table 1) who presented with intermediate uveitis and unusual corneal findings. This patient had a focal area of peripheral corneal oedema 


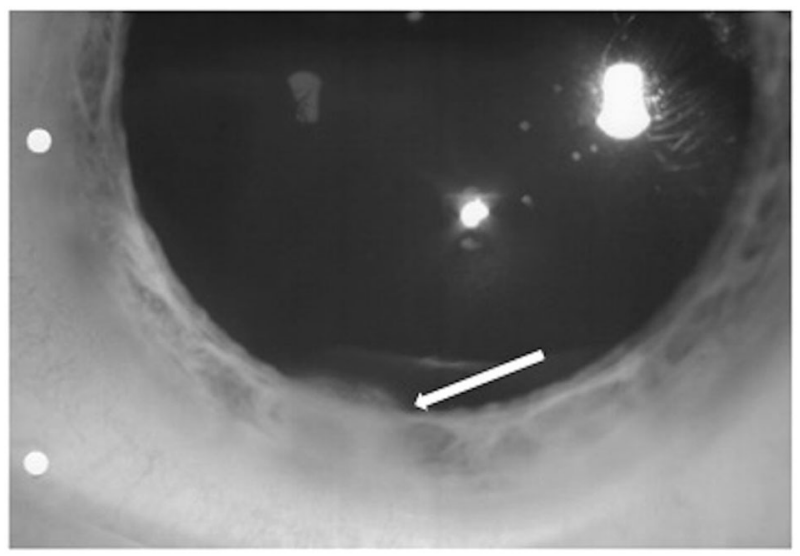

Fig. 1 Photograph of the left cornea from case 5. The white arrow indicates focal area of peripheral corneal endotheliopathy in the setting of PSUS

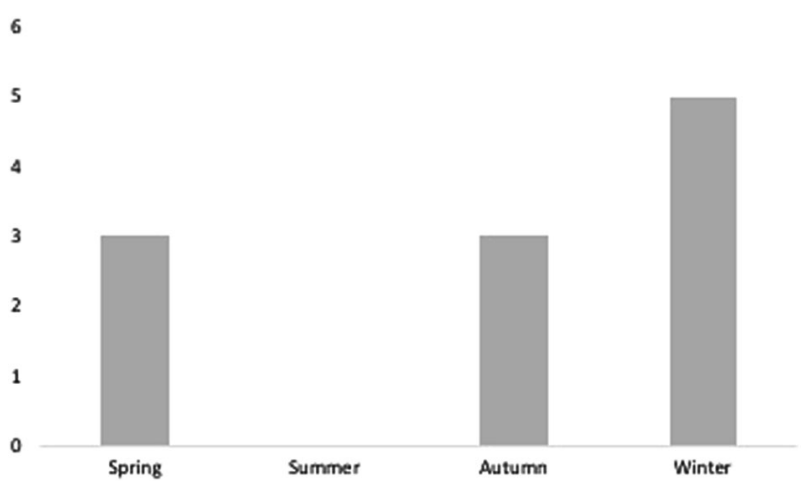

Fig. 2 Variation of presentation of PSUS by season

(Fig. 1) and subsequently developed vitreous haemorrhage as a complication of the intermediate uveitis.

\section{Seasonal preponderance}

Of the patients, $73 \%$ presented in winter or spring. There were no cases of PSUS presenting in the summer months (Fig. 2).

\section{Serological investigations}

The median ASOT was 400 units $/ \mathrm{ml}$. The median AntiDNAse was 500 units/ml.

Both ASOT and Anti-DNase were raised in $45.5 \%$ of patients. Only ASOT was raised in 36.4\%; however, in $27 \%$ of patients, anti-DNase was not tested. Anti-DNase was raised solely in $18.2 \%$ of patients. At presentation, the mean erythrocyte sedimentation rate (ESR) was $16 \mathrm{~mm} / \mathrm{h}$ and the mean C-reactive protein was $2.96 \mathrm{mg} / \mathrm{l}$. Throat swabs were performed in $27 \%$ of patients and was positive in 1 case.
Table 3 Visual outcomes at baseline, 1- and 3-year time points and at final visit

\begin{tabular}{lllll}
\hline & Baseline & 1 Year & 3 Years & Final \\
\hline No. of uveitic eyes & 16 & 14 & 1 & 16 \\
VA $\geq 6 / 12$ & $88 \%$ & $86 \%$ & $0 \%$ & $88 \%$ \\
VA 6/15-6/60 & $12 \%$ & $14 \%$ & $100 \%$ & $12 \%$ \\
VA $\leq 6 / 60$ & $100 \%$ & $100 \%$ & $100 \%$ & $100 \%$ \\
\hline
\end{tabular}

\section{Visual outcome}

Of the 11 patients, 5 had bilateral disease totalling 16 eyes with uveitis. Table 3 outlines the VA at presentation, at 1 - and 3-year time points and at final visit in affected eyes. Of these, $88 \%$ of eyes had a final VA of better than or equal to $6 / 12$ and no eyes were worse than $6 / 60$ at a mean followup of 22 months. Of the patients, $12 \%$ had a VA of less than $6 / 12$ at final visit. The 2 patients with VA less than $6 / 12$ had cystoid macular oedema. At final visit, all patients had a functional VA better than or equal to $6 / 12$ in their better seeing eye when vision in the non-affected eye of unilateral cases was also considered.

\section{Complications}

Complications were known to be present at initial presentation in $64 \%$ of patients. The most common presenting complication was posterior synechiae in $27 \%$ of patients (4 patients). By final visit, complications had occurred in $73 \%$ of patients. Overall, 10 complications were present at initial presentation and 7 complications occurred subsequently. Cystoid macular oedema was seen in 2 patients at presentation and papillitis in 3 patients. Ocular hypertension was present in 1 patient at presentation and was the most common complication to develop during the course of the disease and/or as a result of treatment (6 patients). Cataract (1 patient) and vitreous haemorrhage (1 patient) were also reported as occurring during the course of the disease and/or as a result of treatment.

\section{Systemic medications}

Systemic immunosuppressant treatment was required in $36 \%$ of patients. Methotrexate was the most frequently used systemic immunosuppressant, in $36 \%$ of patients. The median initial dose was $10 \mathrm{mg}$, the median maximum dose $17.5 \mathrm{mg}$ and the median duration of methotrexate treatment was 16 months. All cases were commenced on oral treatment and $75 \%$ of these were subsequently switched to subcutaneous methotrexate to enhance drug bioavailability. Oral steroids were used in $18 \%$ of patients. Biologic agents were used in $18 \%$ of patients. In both cases, adalimumab 
was used at a dose of $40 \mathrm{mg}$ subcutaneously fortnightly. A decision to treat with adalimumab was based on the need for systemic immunosuppression to manage ongoing inflammation in patients with intermediate uveitis who developed steroid-induced ocular hypertension. Both patients had failed on methotrexate and one had also trialled mycophenolate mofetil without remission. The median duration of treatment with adalimumab was 15 months and control of uveitis/remission was achieved in both patients.

\section{Discussion}

Despite numerous cases of PSUS reported since its original description in 1991, it is still an under-recognised condition [2]. In our paediatric uveitis population attending the Belfast Paediatric Uveitis Regional Service, PSU represented $11.6 \%$ of patients [18]. The pathophysiology is not well understood with the mechanism of inflammation unclear. It is thought to represent an immune-mediated reaction where an acute infection can lead to the production of crossreactive tissue-specific antibodies leading to activation of CD4-positive T cells [1]. A serological molecular mimicry exists between group A streptococcal antigens and retinal $\mathrm{S}$ antigen, which may explain the eye's involvement during a host's immunological reaction to the bacterium [19]. With 11 patients in our cohort, we report on the largest series of post-streptococcal uveitis in Caucasian patients.

PSUS can give rise to anterior or posterior uveitis with a full spectrum of clinical features being described. Anterior segment features range from a mild non-granulomatous anterior uveitis to severe granulomatous anterior uveitis $[7,8,12-15]$. Posterior segment features include vitritis, papillitis, cystoid macular oedema (CMO), phlebitis, choroidal lesions, serous retinal detachment and scleritis $[3,7,9,10,12,16]$. One interesting case in our cohort was that of a 9-year-old girl with intermediate uveitis who developed corneal endotheliopathy. This has rarely been described in association with idiopathic intermediate uveitis but not specifically in association with post-streptococcal uveitis [20]. Corneal changes have been reported in PSU associated with group $\mathrm{C}$ streptococcus; however, the case reported by Nataneli et al. [21] described peripheral corneal infiltrates and not corneal oedema. We believe peripheral corneal endotheliopathy is a new finding in PSU.

We demonstrated a seasonal preponderance with $73 \%$ of patients presenting with uveitis in the winter or spring months. More cases occurring during winter months and early spring might be expected when the incidence of group A streptococcal infection is at its highest [22-27]. However, whilst the average interval from infection to the onset of uveitis is 2 weeks, this interval can vary, ranging from 3 days to 3 years after the streptococcal infection [10]. Tinley et al.
[5] expected to find a similar seasonal preponderance in their South African cohort of paediatric patients with PSUS but in fact $50 \%$ of their cases presented in autumn or summer.

Systemic evidence of streptococcal disease is highly suggestive of PSU [4]. When ASOT and anti-DNase are used together, more than $90 \%$ of past streptococcal infections can be correctly identified, with a sensitivity of $95.5 \%$ and specificity of $88.6 \%$ [28]. Some case reports have made the diagnosis of PSUS based on raised ASOT alone $[4,6]$.

The use of systemic suppressants in $36 \%$ of patients in our cohort is comparable to that in the South African series reported by Tinley et al. [5] who used systemic immunosuppressants in $41 \%$ of cases. However, the use of methotrexate was greater in our cohort (36 vs 5\%) and biologic agents in the form of adalimumab were not used in their cohort as compared to $18 \%$ of our patients. The use of adalimumab in PSUS patients has not been previously reported. There is anecdotal evidence to suggest that systemic antibiotics and adenotonsillectomy may have a role in reducing the recurrence of PSUS, but these treatment options were not used in any patients in our series $[9,12,29]$.

Visual prognosis for PSUS patients is good with $88 \%$ of eyes having a VA of 6/12 or better at a mean follow-up of 22 months. The median final VA was $6 / 6$ and ranged from $6 / 4$ to $6 / 19$. This is comparable to the visual outcomes in the case series by Tinley et al. [5] who reported a median VA of $6 / 6$ ranging from $6 / 6$ to $6 / 36$.

We acknowledge the limitations of the retrospective nature of the study. Also, the lack of anti-DNase testing in a small number of cases may reduce the sensitivity and specificity of diagnostic testing. Given the potential for false positives in serological testing and the frequency of streptococcal infections in childhood, it is possible in some instances that the association of streptococcal infection and uveitis in paediatric patients may be coincidental.

However, we adopted the same diagnostic criteria for PSUS employed by Tinley et al. [5] in the largest reported case series of PSUS to date and were careful to exclude other potential causes for paediatric uveitis.

\section{Conclusions}

We report on the largest consecutive series of Caucasian patients under 16 years of age with post-streptococcal uveitis. We have demonstrated a seasonal preponderance with presentation typically in winter or spring. We report a novel finding of corneal endotheliopathy in one of our PSUS patients. We also report on the benefit of adalimumab in the management of severe cases of PSUS; use of biologics in this particular cohort of uveitis patients has not previously been documented. With aggressive treatment and careful monitoring, our patients achieved good visual 
outcomes comparable to other series in the literature for this condition.

\section{Summary}

\section{What was known before:}

- Post-streptococcal uveitis syndrome (PSUS) is still an under-recognised condition. PSUS can give rise to anterior or posterior uveitis with a full spectrum of clinical features being described.

\section{What this study adds:}

- We report on the largest consecutive series of Caucasian patients under 16 years of age with PSUS. We have demonstrated a seasonal preponderance with presentation typically in winter or spring. We report a novel finding of corneal endotheliopathy in one of our PSUS patients. We report on the benefit of adalimumab in the management of severe cases of PSUS.

\section{Compliance with ethical standards}

Conflict of interest The authors declare that they have no conflict of interest.

\section{References}

1. Bisno A. Nonsuppurative poststreptococcal sequelae: rheumatic fever and glomerulonephritis. In: Mandell GL, Bennett JE, Dolin R, editors. Principles and practice of infectious diseases. 6th ed. Philadelphia: Elsevier Churchill Livingstone; 2005. p. 2380-6.

2. Cokington $C$, Han D. Bilateral non-granulomatous uveitis and a post- streptococcal syndrome. Am J Ophthalmol. 1991;112:595-6.

3. De Smet MD. Papillophlebitis and uveitis as a manifestation of post-streptococcal uveitis syndrome. Eye. 2009;23:985-7.

4. Ur Rehman S, Anand S, Reddy A, Backhouse OC, Mohamed M, Mahomed I, et al. Poststreptococcal syndrome uveitis. A descriptive case series and literature review. Ophthalmology. 2006;113:701-6.

5. Tinley C, Van Zyl L, Grötte R. Poststreptococcal syndrome uveitis in South African children. Br J Ophthalmol. 2012;96:87-89.

6. Gallagher MJ, Muqit MMK, Jones D, Gavin M. Poststreptococcal uveitis. Acta Ophthalmol Scand. 2006;84:424-8.

7. Ortiz JM, Kamerling JM, Fischer D, Baxter J. Scleritis, uveitis, and glaucoma in a patient with rheumatic fever. Am J Ophthalmol. 1995;120:538-9.

8. Besada E, Schatz S, Saremi S. Post streptococcal uveitis. Optometry 2000;71:233-8.

9. Besada E, Frauens BJ. Schatz S. Choroiditis, pigment epithelial detachment, and cystoid macular edema as complications of poststreptococcal syndrome. Optom Vis Sci. 2004;81:578-85.
10. Filloy A, Comas C, Català-Mora J. Anterior and intermediate uveitis with retinal vasculitis: an unusual manifestation of poststreptococcal uveitis syndrome. Ocul Immunol Inflamm. 2016;24:607-9.

11. Fretzayas a, Moustaki M, Stefos E, Dermitzaki E, Nicolaidou P. Uveitis: an isolated complication of post-streptococcal syndrome. Ann Trop Paediatr. 2010;30:153-5.

12. Leiba H, Barash J, Pollack A. Poststreptococcal uveitis. Am J Ophthalmol. 1998;126:317-8.

13. Benjamin A, Tufail A, Holland GN. Uveitis as the only clinical manifestation of poststreptococcal syndrome. Am J Ophthalmol. 1997;123:258-60.

14. Kobayashi S, Tamura N, Ikeda M, Sakuraba K, Matsumoto T, Hashimoto H. Uveitis in adult patients with poststreptococcal reactive arthritis: the first two cases reported associated with uveitis. Clin Rheumatol. 2002;21:533-5.

15. Holland GN. Recurrent anterior uveitis associated with streptococcal pharyngitis in a patient with a history of poststreptococcal syndrome. Am J Ophthalmol. 1999;127:346-7.

16. Hall AJH, Barton K, Watson PG, Lightman S. Scleritis in association with poststreptococcal vasculitis. Arch Ophthalmol. 1993;111:1324-5.

17. Jabs DA. Standardization of uveitis nomenclature for reporting clinical data. Results of the first international workshop. Am J Ophthalmol. 2005;140:509-16.

18. Curragh DS, O’Neill M, McAvoy CE, Rooney M, McLoone E. Pediatric uveitis in a well-defined population: improved outcomes with immunosuppressive therapy. Ocul Immunol Inflamm. 2018;26:978-85.

19. Cunningham MW. Pathogenesis of group A streptococcal infections. Clin Microbiol Rev. 2000;13:470-511.

20. Tugal-Tutkun I. Pediatric uveitis. J Ophthalmic Vis Res. 2011;6:259-69.

21. Nataneli N, Aguilera ZP, Rosenbaum PS, Goldstein T, Mayers M. Poststreptococcal keratouveitis associated with group C streptococcus pharyngitis. Clin Ophthalmol. 2011;5:1257-9.

22. Olafsdottir LB, Erlendsdóttir H, Melo-Cristino J. et al. nvasive infections due to streptococcus Pyogenes: seasonal variation of severity and clinical characteristics, Iceland, 1975 to 2012. Euro Surveill. 2014;19:5-14.

23. Luca-Harari B, Darenberg J, Neal S, et al. Clinical and microbiological characteristics of severe Streptococcus pyogenes disease in Europe. J Clin Microbiol. 2009;47:1155-65.

24. Lamagni TL, Darenberg J, Luca-Harari B, et al. Epidemiology of severe Streptococcus pyogenes disease in Europe. J Clin Microbiol. 2008;46:2359-67.

25. O'Loughlin RE, Roberson A, Cieslak PR, et al. The epidemiology of invasive group A streptococcal infection and potential vaccine implications: United States, 2000-4. Clin Infect Dis. 2007;45:853-62.

26. Kaplan EL, Rothermel CD, Johnson DR. Antistreptolysin O and anti-deoxyribonuclease B titers: normal values for children ages 2 to 12 in the United States. Pediatrics. 1998;101(1 Pt 1):86-88.

27. Gunnlaugsson S, Kristinsson KG, Steingrimsson O. [Results of cultures and serotyping of S. pyogenes 1986-93.]. Laeknabladid. 1995;81:728-32.

28. Blyth CC, Robertson PW. Anti-streptococcal antibodies in the diagnosis of acute and post-streptococcal disease: streptokinase versus streptolysin $\mathrm{O}$ and deoxyribonuclease B. Pathology. 2006;38:152-6.

29. Ovchinsky A, Schulman S, Rosenfeld RM. Adenotonsillectomy as a treatment option for poststreptococcal uveitis. Laryngoscope. 2002;112:658-60. 